\title{
A head-mounted display-based personal integrated-image monitoring system for transurethral resection of the prostate
}

\author{
Soichiro Yoshida, Kazunori Kihara, Hideki Takeshita, Yasuhisa Fujii \\ Tokyo Medical and Dental University Graduate School, Tokyo, Japan
}

Videosurgery Miniinv 2014; 9 (4): 644-649

DOI: $10.5114 /$ wiitm.2014.43040

\begin{abstract}
The head-mounted display (HMD) is a new image monitoring system. We developed the Personal Integrated-image Monitoring System (PIM System) using the HMD (HMZ-T2, Sony Corporation, Tokyo, Japan) in combination with video splitters and multiplexers as a surgical guide system for transurethral resection of the prostate (TURP). The imaging information obtained from the cystoscope, the transurethral ultrasonography (TRUS), the video camera attached to the HMD, and the patient's vital signs monitor were split and integrated by the PIM System and a composite image was displayed by the HMD using a four-split screen technique. Wearing the HMD, the lead surgeon and the assistant could simultaneously and continuously monitor the same information displayed by the HMD in an ergonomically efficient posture. Each participant could independently rearrange the images comprising the composite image depending on the engaging step. Two benign prostatic hyperplasia (BPH) patients underwent TURP performed by surgeons guided with this system. In both cases, the TURP procedure was successfully performed, and their postoperative clinical courses had no remarkable unfavorable events. During the procedure, none of the participants experienced any HMD-wear related adverse effects or reported any discomfort.
\end{abstract}

Key words: data display, cystoscopes, prostatic hyperplasia, transurethral resection of prostate.

\section{Introduction}

Recently, new minimally invasive techniques have been introduced to endourological procedures $[1,2]$. The introduction of such technological advances as bipolar electrodes and high-definition camera systems have improved the results of transurethral resection of the prostate (TURP) [3]. However, due to possible intraoperative complications of the TURP procedure, extreme care should be exercised to prevent bleeding, perforation of the prostatic capsule, and injury of the urethral sphincter. Using intraoperative transrectal ultrasonography (TRUS) investigation to guide TURP is another way to improve the treatment efficacy and to minimize the intraoperative risks. The usefulness of intraoperative TRUS as a navigation system for TURP has been reported [4]. Furthermore, monitoring vital signs is also useful for early identification of these possible complications. Therefore, it would be ideal if all physicians involved in performing TURP constantly and simultaneously could monitor all of this information during the operation.

The application of a head-mounted display (HMD) as a new imaging monitor has been proposed for anesthesia management and laparoscopic surgery [5-7]. The quality of modern HMDs has been dramatically improved in terms of resolution, wearability, and weight. The HMZ-T2 (Sony Corporation, Tokyo, Japan) is a 330-g binocular HMD composed of 0.7 -inch organic light-emitting diode (OLED) screens

\section{Address for correspondence}

Prof. Kazunori Kihara MD, PhD, Tokyo Medical and Dental University Graduate School, 1-5-45, Yushima, Bunkyo-ku, 113-8519 Tokyo, Japan, phone: +8135803 5295, fax: +8135803 5295, e-mail: k-kihara.uro@tmd.ac.jp 
(resolution: 1,280 $\times 720$ pixels) for each eye, which provides the wearer with sharp and high-contrast images. We have applied HMD as an imaging monitor for gasless single-port radical nephrectomy [8]. The excellence of the HMD as a personal imaging monitoring system led us to apply the HMD system to urological endoscopic surgery. We developed a Personal Integrated-image Monitoring System, the PIM System, by combining video splitters and multiplexers with the HMD system. In this system, multiple forms of information can be seamlessly integrated and displayed by the HMD. This system allows multiple participants to simultaneously view the same images. Furthermore, each participant can independently rearrange the images comprising the composite image displayed on his HMD depending on the engaging step of the surgery. We present here an introduction of the PIM System for TURP.

\section{Case report}

Two urologists (one lead surgeon and one assistant) performed TURP using the PIM System in $2 \mathrm{BPH}$ cases. The age and the estimated prostate volume of the first case were 64 years and $57 \mathrm{~g}$, and those of the second case were 75 years and $68 \mathrm{~g}$. The lead surgeon and the assistant each wore an HMD throughout the procedure. Informed consent was obtained following a complete description of the TURP procedure performed under the guidance of TRUS using the PIM System, and the procedure was carried out with the approval of our university's ethics committee.

Transurethral resection of the prostate was performed using a bipolar transurethral resection in a saline system (TURis, Olympus, Tokyo, Japan). The intraoperative TRUS evaluation was performed using an ultrasound machine (HI VISION Preirus, Hitachi-Aloka, Tokyo, Japan) and a b-mode biplane TRUS probe (3-7 MHz; EUP-U533, Hitachi-Aloka). The imaging information obtained from the cystoscope, the TRUS, the video camera attached on the HMD (HMD-mounted camera), and the patient's vital monitor were split using an imaging splitter (400VGA003, Sanwa Supply Incorporated, Okayama, Japan) and the composite image was output into 2 multiplexers (VPM-H1, MEDIAEDGE Corporation, Hyogo, Japan). The images were integrated using a four-split screen technique on the multiplexer and were displayed on the HMDs (HMZ-T2) (Figure 1).
Under lumbar anesthesia, the standard TURP was performed under the TRUS guide using the PIM System. The assistant manipulated the TRUS placed in the patient's rectum during all the stages of the TURP (Photo 1).

The lead surgeon and the assistant could simultaneously and continuously monitor the integrated multiple images displayed by the HMD in an ergonomically efficient posture. The images displayed by the four-split screens had enough resolution for clinical application in this surgical procedure. In handling

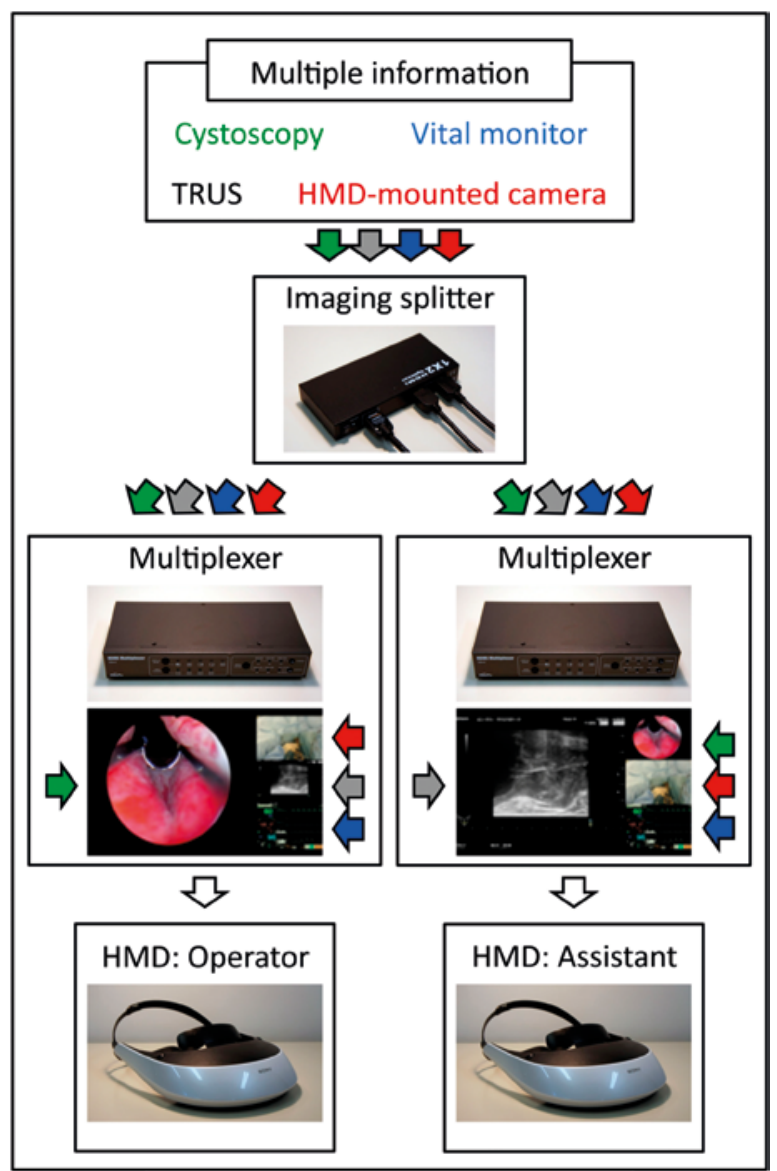

Figure 1. Overview of the personal integratedimage monitoring system. Multiple input information is split using an imaging splitter, and integrated into one composite image using a multiplexer. Each wearer of the head-mounted display (HMD) can independently arrange the array of the displayed images. In this figure, each type of input information is color coded: cystoscopy, green; transrectal ultrasonography, (TRUS), black; vital signs monitor, blue; HMD-mounted camera, red 


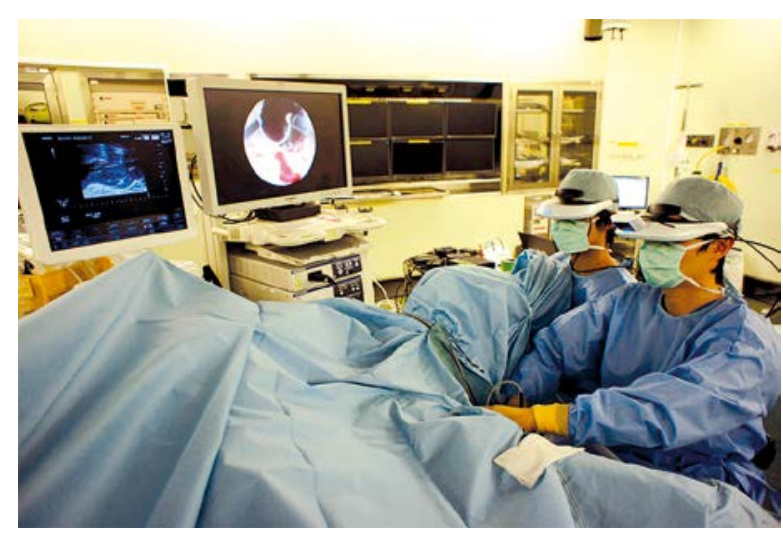

Photo 1. Photograph of transurethral resection of the prostate being performed using the personal integrated-image monitoring system paired with transrectal ultrasonography. Both the lead surgeon and the assistant wear a head-mounted display during all stages of the procedure

the surgical tools, the HMD wearer could use either the view from a front-viewing camera attached to the HMD or direct vision by moving his angle of sight downward.

In the 2 cases we report here, the lead surgeon opted to arrange the cystoscopic view as the main image displayed by the HMD during the resection of prostatic adenoma, and the assistant chose the ultrasonography view as the main image (Photo 2). When viewing the TRUS images, it was easy to identify the landmarks for TURP; the contour of the prostate, the shape of protruding adenoma in the bladder and the shape of the apex were clearly visible (Photo 2). During the resection of the prostate, the TRUS guide enabled the surgeon to recognize the amount of the residual adenoma and the position of the rectum and bladder. The resectoscope hampered the visibility of the anterior part of the prostate (Photo 2). After the resection was finished, TRUS investigation clearly depicted the prostatic bed (Photo 3). Transrectal ultrasonography clearly depicted the resected prostatic tips in the bladder during bladder evacuation. The operator was able to monitor the position of the bladder evacuator, thus avoiding accidental damage to the bladder wall. Furthermore, TRUS navigated the Foley catheter to the proper position.

In both cases, the TURP procedure was successfully performed, and there were no notable negative outcomes or incidents in the postoperative course of either patient. The resected volume was $30 \mathrm{~g}$ in the first case and $42 \mathrm{~g}$ in the second case. During the procedure, neither the lead surgeon nor the assistant experienced any HMD-related adverse effects or reported any discomfort.

\section{Discussion}

We performed an assessment of an HMD-based personal image integrating system for its utility in the TURP procedure. We found that this new system, which we have called the PIM System, was both safe and useful for performing the TURP procedure. Because both the lead surgeon and the assistant wore an HMD, they could share the same information and independently arrange their image arrays, depending upon their roles and steps of the surgery, and they could do so in an ergonomically efficient manner. Compared with conventional TURP performed solely with endoscopic visual cues, TURP using the PIM System as a surgical guide system is expected to improve the efficiency and safety of the procedure. However, we need further studies to prove the actual benefits of this system.

This is the first study to use an HMD system in TURP. The proposed concept in this paper is novel in terms of the application of HMD as a personalized surgical guide system. Because TRUS can clearly visualize the prostate shape and its anatomical relationship to adjacent structures, Hung et al. [9] and van der Poel et al. [10] used TRUS investigation as a surgical navigation technique for radical prostatectomy to identify anatomical landmarks during the surgery. In transurethral endoscopic surgery, it is also possible to use the rectum as a "window" to monitor the surgical field using TRUS in a simple way. Furthermore, by using an imaging splitter, one can output the images into multiple HMD, as many as needed, depending on the number of participants in the surgery. In this way, all participants, including the anesthesiologist and even students, can share the same information with the lead surgeon using the PIM System.

Our PIM System is composed of HMD, imaging splitters and multiplexers. All of these components are commercially available at low cost. In addition to the compact size of the system, this system is attractive in that it can be applied to many procedures because one can arrange the system components and select the input information at will, changing the number of splitters and multiplexers, and selecting the input images depending on the required information. 

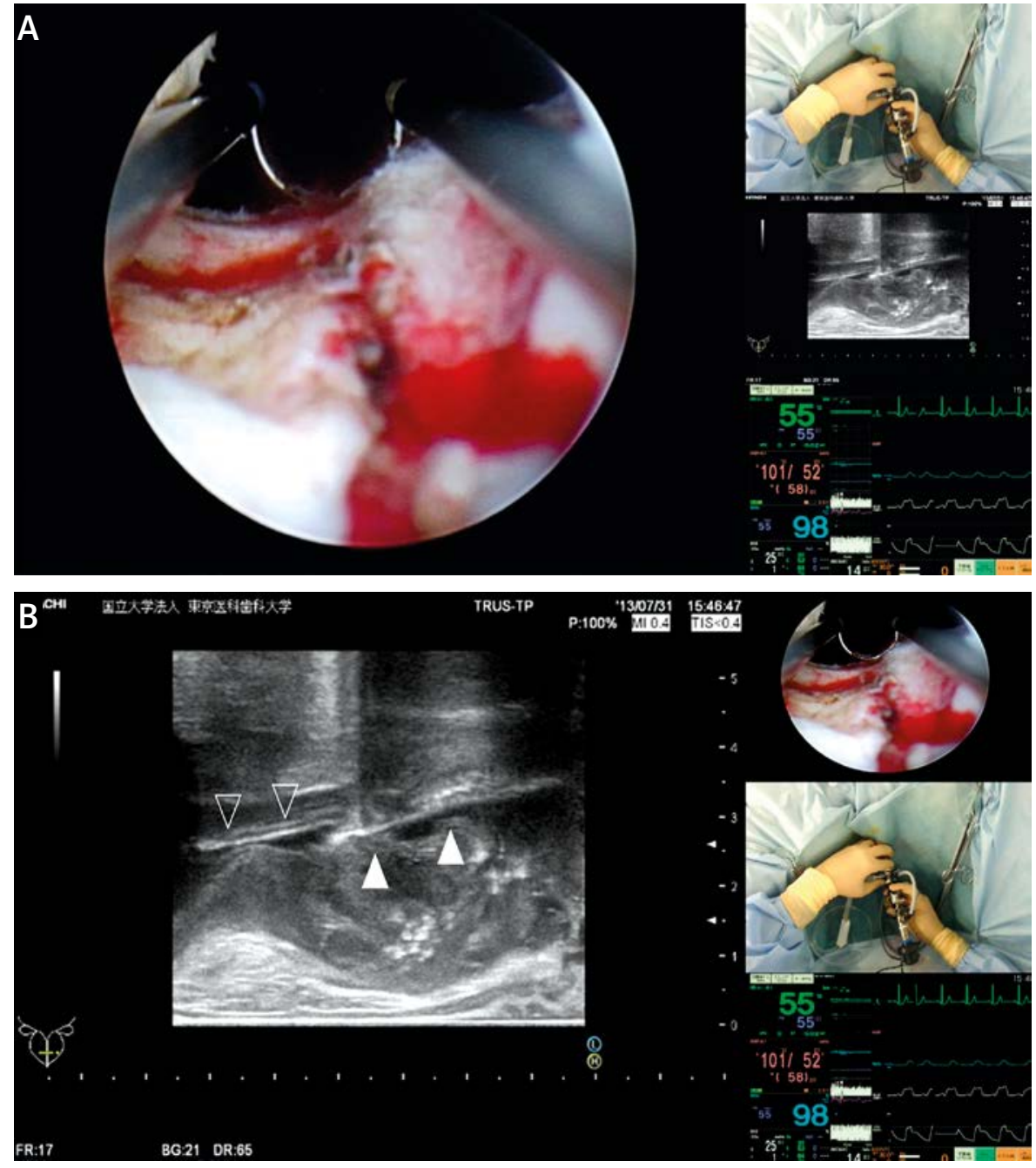

Photo 2. Captured image of displayed integrated image data including the cystoscopic image, the transrectal ultrasonographic image, the patient's vital signs, and the view from a head-mounted camera attached on the head-mounted display. A - Composite image using the endoscopic image as the primary image. B - An arrangement using the ultrasonographic image as the primary image. On transrectal ultrasonography, the resectoscope (arrow heads) and the resection loop (white-outlined arrowheads) are clearly visible as a hyperechoic lines. However, the resectoscope hampered the visibility of the anterior part of the prostate

In the current study, because the assistant handled the TRUS transducer, two urologists participated in each operation. Recently, the utility of a robotic TRUS probe holder (R-TRUS) was reported [9]. We are now planning to combine the R-TRUS with our PIM system in further research. The four-split screen view of the HMD limits the display space for each split image. However, the high-resolution OLED HMZ-T2 display provides the physician with high-contrast and sharp quality images, which can overcome this limitation.

\section{Conclusions}

Herein, we showed the utility of the PIM System as a surgical guide system for TURP. Although further study is needed to prove the actual benefits of this new system, we think that the PIM System is a promising surgical guide system for all urological transurethral surgery, including TURP. 

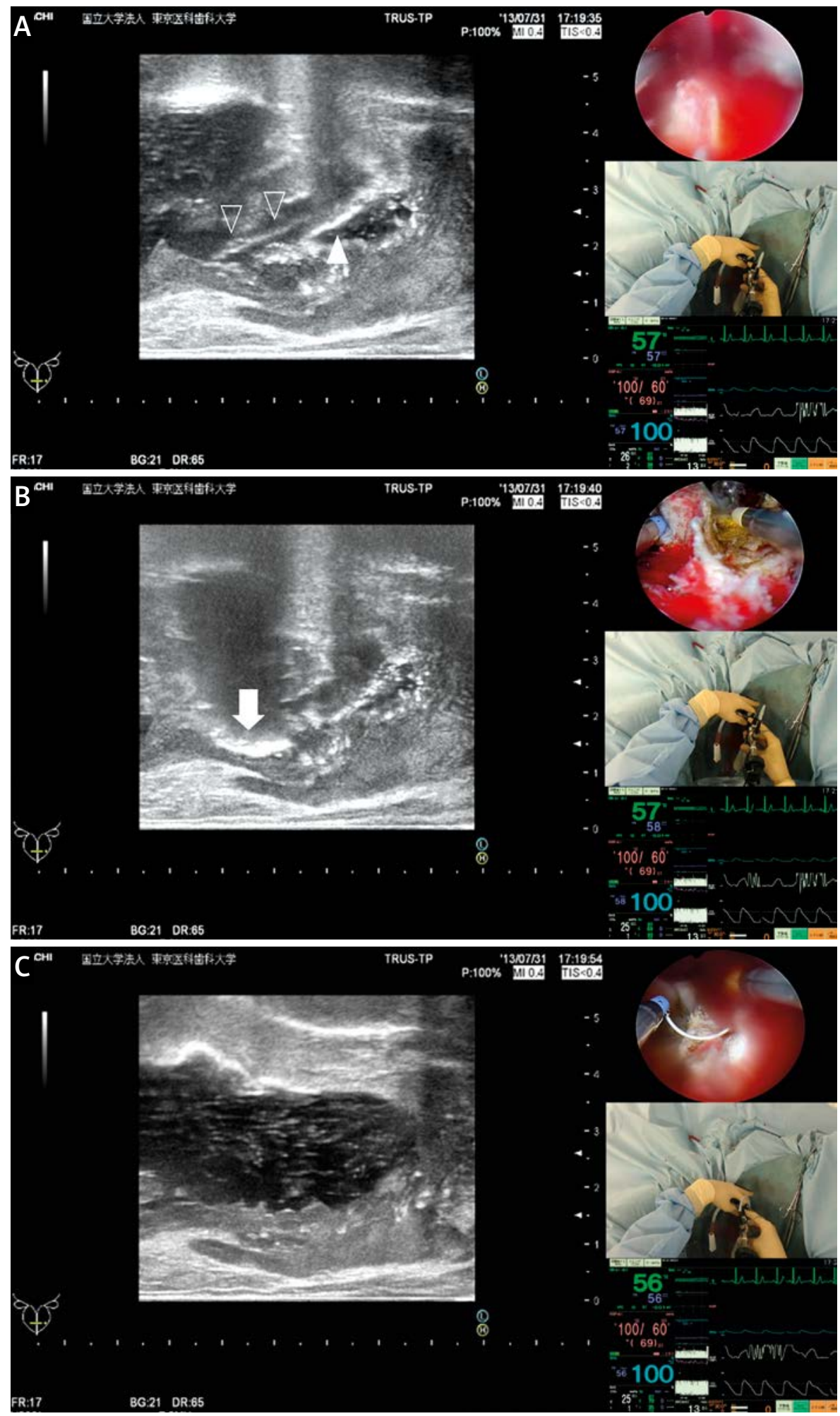

Photo 3. Captured images of the displayed data in the head-mounted display worn by the lead surgeon (A) and the assistant $(\mathrm{B}, \mathrm{C})$ during the resection of the posterior part of the prostate. On transrectal ultrasonography, the resectoscope (arrow heads) and the resection loop (white-outlined arrowheads) are clearly visible as a hyperechoic lines. The resected tissue is visible as a hyperechoic signal just after the electrical resection (arrow). After the resection was finished, the prostatic bed was clearly visible on transrectal ultrasonography $(\mathrm{C})$ 


\section{References}

1. Czudek S. Robotic surgery - a taste of Hollywood? Videosurgery Miniinv 2013; 8: 95-8.

2. Roslan M, Markuszewski MM, Bagińska J, Krajka K. Suprapubic transvesical laparoendoscopic single-site surgery for vesicovaginal fistula repair: a case report. Videosurgery Miniinv 2012; 7: 307-10.

3. Oelke M, Bachmann A, Descazeaud A, et al. EAU guidelines on the treatment and follow-up of non-neurogenic male lower urinary tract symptoms including benign prostatic obstruction. Eur Urol 2013; 64: 118-40.

4. Aliaev lu G, Grigorian VA, Tsarichenko DG, et al. Transurethral electroresection of prostatic adenoma under transrectal ultrasonic control. Urologiia (Moscow, Russia: 1999) 2006; 3: 8-12.

5. Liu D, Jenkins SA, Sanderson PM, et al. Monitoring with head-mounted displays in general anesthesia: a clinical evaluation in the operating room. Anesth Analg 2010; 110: 1032-8.

6. Liu D, Jenkins SA, Sanderson PM, et al. Monitoring with head-mounted displays: performance and safety in a fullscale simulator and part-task trainer. Anesth Analg 2009; 109: 1135-46.

7. Prendergast CJ, Ryder BA, Abodeely A, et al. Surgical performance with head-mounted displays in laparoscopic surgery. J Laparoendosc Adv Surg Tech A 2009; 19 Suppl. 1: S237-40.

8. Kihara K, Fujii Y, Masuda H, et al. New three-dimensional head-mounted display system, TMDU-S-3D system, for minimally invasive surgery application: procedures for gasless single-port radical nephrectomy. Int J Urology 2012; 19: 886-9.

9. Hung AJ, Abreu AL, Shoji S, et al. Robotic transrectal ultrasonography during robot-assisted radical prostatectomy. Eur Urol 2012; 62: 341-8.

10. van der Poel HG, de Blok W, Bex A, et al. Peroperative transrec tal ultrasonography-guided bladder neck dissection eases the learning of robot-assisted laparoscopic prostatectomy. BJU Int 2008; 102: 849-52

Received: 9.10.2013, accepted: 23.01.2014. 